www.nature.com/clinicalpractice/cardio

\section{Inhibition of soluble epoxide hydrolases as a new treatment target for cardiac hypertrophy}

Cardiac failure often results from sustained cardiac hypertrophy. The transcription factor nuclear factor kappa $\mathrm{B}$ (NFkB) regulates the expression of genes involved in cellular response to stress and has previously been implicated in the development of cardiac hypertrophy. Soluble epoxide hydrolase (sEH) inhibitors increase levels of endogenous lipid epoxides such as epoxyeicosatrienoic acids, which can interfere with NFKB-mediated gene transcription. Further investigations into this process in mice have lead Xu et al. to present sEH inhibitors as a possible novel treatment for cardiac hypertrophy.

Using a murine model of pressure-induced cardiac hypertrophy, the authors demonstrated that various $\mathrm{sEH}$ inhibitors could both prevent and reverse cardiac hypertrophy through inhibition of the NFKB pathway, preventing the progression of cardiac deterioration. The exact mechanism of the inhibition remained unclear, indicating a need for further study. Experiments with a primary culture of neonatal mouse cardiomyocytes confirmed that sEH inhibitors had concentration-dependent antihypertrophic effects, blocking NFKB activation. Furthermore, in vivo electrophysiologic recordings showed that $\mathrm{sEH}$ inhibitors had a role in the prevention of cardiac arrhythmias, the risk of which is greatly increased following cardiac hypertrophy. The authors believe that SEH inhibitors could provide a costeffective, efficacious method for preventing ventricular arrhythmias associated with cardiac hypertrophy.

Original article Xu D et al. (2006) Prevention and reversal of cardiac hypertrophy by soluble epoxide hydrolase inhibitors. Proc Natl Acad Sci USA 103: 18733-18738

\section{Myocardial perfusion imaging and multislice CT are complementary in CAD detection}

Myocardial perfusion imaging (MPI) and multislice CT (MSCT) both enable noninvasive imaging of suspected coronary artery disease (CAD). The former detects ischemia, whereas the latter identifies atherosclerosis through visualization of coronary artery stenosis. A comparison of these techniques shows that they provide complementary information in patients with an intermediate likelihood of CAD, indicating that the methods might best be used in combination.

Both MPI and MSCT were performed in 114 patients who had presented to outpatient clinics with chest pain and no history of CAD. Of the 41 (36\%) patients with normal MSCT findings, $37(90 \%)$ also had normal MPI results. Over half of the 73 patients with abnormal MSCT findings, however-including those with obstructive CAD-had normal MPI findings. Likewise, approximately half of the patients with normal MPI results had abnormal MSCT findings. Importantly, these data indicate that the degree of stenosis doesn't necessarily reflect the hemodynamic significance of a lesion. A subcomparison of MSCT with invasive coronary angiography showed good correlation of results, inferring that MSCT has high diagnostic accuracy in individuals with an intermediate likelihood of CAD.

How MPI and MSCT can best be combined in the clinical setting is unclear, say the authors. They suggest, however, that MSCT could be used as a first-line test, with patients undergoing further imaging if the results are positive in order to determine the hemodynamic significance of the lesion.

Original article Schuijf JD et al. (2006) Relationship between noninvasive coronary angiography with multi-slice computed tomography and myocardial perfusion imaging.

J Am Coll Cardiol 48: 2508-2514 